\title{
O Uso de Fitoterápicos na Redução e no Tratamento de Hipertensão Arterial Sistêmica \\ Elisangela de Meira ${ }^{1}$, Edirléia de Fatima Kloster ${ }^{1}$, Josieli Maria Kosak ${ }^{1}$, Mayara Francini Kolitski ${ }^{1}$, Vinicius Bastos Cutilaki ${ }^{1}$, Caryna Eurich Mazur ${ }^{2}$
}

\begin{abstract}
Resumo: Buscou-se analisar o efeito de alguns fitoterápicos na prevenção e tratamento de doenças cardiovasculares, em especial na hipertensão arterial sistêmica. Foi realizado uma revisão de literatura na biblioteca virtual LILACS, Scielo, Google Acadêmico, e Pubmed, referentes aos anos de 1990 a 2016. Foram encontrados e descritos 5 fitoterápicos. A fitoterapia é muito utilizada, porém muito sem fundos científicos, desta forma mais estudos são necessários para comprovação da eficiência dos mesmos.
\end{abstract}

Palavras-chave: Fitoterapia, Prevenção De Doenças, Hipertensão.

\section{The Herbal use in Reducing and Tratament of Hypertension}

\begin{abstract}
To examine the effect of some herbal remedies in the prevention and treatment of cardiovascular diseases, especially in hypertension. We performed a literature review in the virtual library LILACS, Scielo, Google Scholar and Pubmed for the years 1990 to 2016. Results and Discussion: found and described 5 herbal medicines. The herbal medicine is widely used but many without scientific backgrounds, thus more studies are needed to prove the efficiency.
\end{abstract}

Key words: Herbal Medicine, Disease Prevention, Hypertension.

\section{Introdução}

A hipertensão, uma das doenças mais prevalentes em todo o mundo, é uma doença crônica caracterizada pela pressão sanguínea elevada. Está associada a vários fatores de risco, como acidentes vasculares cerebrais, doenças cardíacas, insuficiência renal e aneurisma (GUYTON; HALL, 2006). Existem muitos fatores que podem causar hipertensão, tais como estilo de vida os hábitos alimentares, sedentarismo, o estresse, a obesidade visceral, a idade entre outros (MAHAN; STUMP, 2011).

Devido à alta prevalência de hipertensão e doenças cardíacas, a Organização Mundial da Saúde (OMS), tem estimulado o desenvolvimento de políticas públicas que priorizam a utilização de plantas medicinais no tratamento de doenças, e enfatiza a necessidade de novas pesquisas feitas com plantas e produtos naturais (MARMITT et al., 2016).

\footnotetext{
${ }^{1}$ Nutricionista. Faculdade Campo Real. Guarapuava, Paraná, Brasil. Contato: meira_elisangela@ @otmail.com

${ }^{2}$ Nutricionista. Mestre em Segurança Alimentar e Nutricional. Docente do curso de Nutrição. Faculdade Campo Real. Guarapuava, Paraná, Brasil. E-mail: carynanutricionista@gmail.com
} 
Seguindo as recomendações da OMS e buscandoestimular mecanismos naturais de prevenção de agravos e recuperação da saúde e estimular vinculo do ser humano com o meio ambiente e sociedade o ministério da saúde brasileiro aprovou a Política Nacional de Praticas Integrativas e Complementares (PNPIC) que comtempla dentre outras a área de plantas medi cinais e fitoterapiaque é caracterizada pelo uso de plantas medicinais na sua totalidade de origem vegetal e em suas diferentes formas farmacêuticas (BRASIL, 2006).

Desta forma o presente estudo tem como objetivo analisar o efeito de alguns fitoterápicos na redução e tratamento de doenças cardiovasculares, em especial na hipertensão arterial sistêmica.

\section{Metodologia}

O presente estudo trata-se de uma revisão bibliográfica acerca dos fitoterápicos com propriedades anti-hipertensivas. Para obtenção dos dados foi utilizada como base de dados periódicos eletrônicos: Scielo, Google Acadêmico, Lilacs e Pubmed. Abordando os seguintes temas, fitoterápicos e hipertensão, fitoterápicos hipotensores, plantas medicinais e pressão arterial sistêmica, devido à falta de estudos optou-se por abordar um período mais longo dos anos de 1990 até 2016 além de englobar artigos em inglês, espanhol e em português.

\section{Resultados e discussão}

\section{Alho (Allium sativum)}

O alho (Allium sativum) é utilizado há séculos, devido seus benefícios a saúde, prevenindo doenças cardíacas e infecções. O uso medicinal do alho vem desde a época dos faraós egípcios até a Segunda Guerra Mundial. Durante a Segunda Guerra Mundial o caldo que era extraído dessa raiz era muito utilizado na cicatrização de ferimentos devido ao seu efeito antibiótico, nesta época era chamado de "penicilina russa" (TALBOTT; HUGHES, 
2015). O alho é benéfico a saúde cardiovascular devido sua atividade anti-hipertensiva, inibição da agregação plaquetária, e redução do colesterol (LAMARÃO; NAVARRO, 2012).

O alho possui em sua composição o enxofre que é responsável pelo efeito cardioprotetor, e entre outros compostos com: aliina, alicina, S-alil-cisteína e S-metilcisteína. Essa formulação também pode ser encontrada na cebolinha, alho-poró e cebola, porém, o alho é o que possui maior quantidade dessas substâncias. Esses elementospodem reduzir os níveis de colesterol sanguíneo, protegem as células endoteliais vasculares contra lesões, devido a redução do estresse oxidativo e a inibição da oxidação do LDL-c, também possuem a função antitrombótica por meio do aumento da atividade fibrinolítica e da redução da agregação plaquetária e promove redução da pressão arterial devido ao efeito de relaxamento das células lisas, causando vasodilatação (TALBOTT; HUGHES, 2015).

É recomendado que o alho seja consumido na forma crua, pois durante o processo de trituração e cocção ele perde quase todas as suas propriedades benéficas a saúde (ALMEIDA; SUYENAGA, 2009)

Com relação a recomendação diária de alho, ainda não existe um consenso, porém a American Dietetic Association recomenda o consumo de 600-900 mg/dia, ou seja, o equivalente a 1 dente de alho cru por dia para obter de seus benefícios (LAMARÃO; NAVARRO, 2012).

São raras as reações adversas que podem ocorrer devido ao consumo de alho, porém há relatos de manifestações gastrointestinais leves, como pirose e náuseas. Em alguns casos, o consumo muito elevado de alho pode potencializar o efeito do ácido-acetilsalicílico, da vitamina E e do óleo de peixe (TALBOTT; HUGHES, 2015).

\section{Alpinia zerumbet (Pers.) B. L.}

A Alpinia zerumbet é uma planta originária da Ásia, da família Zingiberaceae, muito cultivada pela beleza de suas flores, é encontrada em abundancia no nordeste do Brasil onde é conhecida popularmente como colônia, paco-seroca, cuité-açu, pacová, gengibre-concha, cardamomo-do-mato, cardamomo-falso, cana-do-brejo, cana do mato e paco-seroso (MENDONÇA et al., 1991). É uma planta herbácea que chega a atingir 2,5 metros de altura, 
apresentando folhas curtas e lanceoladas, com caules aéreos e agrupados em touceiras (ALBUQUERQUE; NEVES, 2004).

O efeito hipotensor e levemente diurético está entre as principais propriedades farmacológicas da A. zerumbet, obtidos através do chá e do óleo das folhas (MENDONÇA et al., 1991). Em estudo de Mpalantinos et al. (1998) com o extrato aquoso da planta identificaram rutina, campferol-3-O-rutinosideo, campferol-3-O-glucorunido, catequina e epicatequina, flavonoides com que contribuem para a atividade hipotensora e diurética da $A$. zerumbet. Em estudo com pacientes portadores de hipertensão arterial em estágio leve a moderado, Lordelo et al. (2000), administraram folhas secas pulverizadas e encapsuladas de A. zerumbet, e concluíram que as cápsulas foram seguras e eficazes no controle da hipertensão dos pacientes, justificando a eficácia devido aos flavonoides presentes na planta.

Emiliano (2002) investigando o efeito do extrato hidro alcoólico da A. zerumbet, concluiu em seus estudos que o efeito hipotensivo do extrato hidroalcoólico do A. zerumbet é devido ao efeito vasodilatador que exerce no leito vascular mesentérico com consequente diminuição da resistência vascular periférica.

Lahlou et al. (2003) observaram que o tratamento intravenoso com óleos essenciais da A. zerumbetem ratos anestesiados por pentobarbitona, levava a hipotensão imediata, podendo ser atribuída ao terpineno-4-ol substância presente tanto nas folhas quanto no rizoma da planta. O uso da Alpinia zerumbet não em recomendado para gestantes, lactantes, lactentes, crianças menores de dois anos, alcoolistas e diabéticos. Pois no tratamento com o extrato hidro alcoólico foi observado o aumento de transaminases e HDL-c, e não foram totalmente elucidados os efeitos de todos os seus componentes (MENDONÇA et al., 1991).

\section{Cavalinha (Equisetum arvense)}

A Equisetum arvense, popularmente conhecida como Cavalinha (ou rabo-decavalo, lixa-vegetal, erva-carnuda) é uma planta da família das Equisetaceas, originária da Europa. Trata-se de uma herbácea perene, de rizoma horizontal, sobre o qual se desenvolvem dois tipos de caules aéreos; os férteis, que são acinzentados, nascem no final do inverno e terminam em espigas de 10 a $20 \mathrm{~cm}$ de altura e os estéreis, que são verdes, de 20 a $80 \mathrm{~cm}$ de 
altura, aparecem no verão e secam no inverno. Ambos são finos, ocos, em formato de cana e articulados. (NOGUEIRA JUNIOR, 2014). É nativo das áreas pantanosas de todo o Brasil, sendo frequentemente cultivado com fins ornamentais em lagos decorativos (LORENZI, 2008).

No estudo de Mello e Budel (2014), em análise da literatura sobre espécies de Equisetum encontrados no Brasil com propriedades farmacológicas, demostrou que as espécies de Equisetum são utilizadas popularmente como diuréticas, anti-inflamatórias, cicatrizantes, digestivas, hipoglicemiantes, remineralizante, hipotensora e antioxidante diurético, digestivo e antianêmico.

Toma-se sob forma de infusão, colocando $5 \mathrm{~g}$ do caule (aproximadamente 1 colher de sopa) em $100 \mathrm{ml}$ de água fervente, por 10 minutos. Deixa-se descansando abafada por 15 minutos e côa para beber. Orienta-se beber de 1 a 3 xícaras de chá ao dia (NOGUEIRA JUNIOR, 2014).

\section{Pitangueira (Eugenia Uniflora L.)}

A Eugenia uniflora L. conhecida como pitangueira, pertence à família das Myrtaceae, tem seus frutos muito conhecidos e consumidos, visto que é uma planta nativa brasileira e possui um grande potencial econômico com sua comercialização (BEZERRA et al., 2004). Dentre as espécies, é encontrado na forma de arbusto e de árvores, sua altura pode chegar de três a doze metros e possuir frutos de diversas colorações como amarelo, alaranjado, vermelho e preto (DE QUEIROZ, 2015).

Tendo em sua composição alguns compostos fenólicos de ações antioxidante, hipoglicemiante, antirreumáticas podendo ser usadas em caos de problemas gástricos e como agente hipotensivo (ALMEIDA et al., 1995). Estudos mostram que extratos da folha obtidos de plantas do gênero Eugenia apresentam diversas ações, sendo as principais antibacterianas (PESSINI, 2003), antifúngicas (SOUZA 2002; COSTA, 2010) e antioxidante (MAGINA, 2010).

Outro efeito obtido com sua utilização foi anti-hipertensiva. Em seu estudo, Consolini, Baldini e Amat (1999), verificaram que o extrato de folhas de Eugenia uniflora, teve uma 
ação hipotensora, vasodilatadora e diurética quandotestado em ratos, os autores concluíram que essa ação pode estar relacionada com um aumento do fluxo sanguíneo renal. Cirqueira (2005) confirmou em seu estudo uma ação hipotensora e diurética com uso de Eugenia uniflora L. em ratos, onde utilizou de 56 a $172 \mathrm{mg} / \mathrm{kg}$ de peso de extrato seco de pitanga obtido através da evaporação do extrato aquoso, obtendo um pico de diurese na concentração de $94 \mathrm{mg} / \mathrm{kg}$.

Mesmo que seja popularmente utilizada há muito tempo a Eugenia uniflora L., ainda necessitam mais evidências cientificas no que tange seus principais efeitos, utilização de forma terapêutica, bem como posologia e toxicidade. Visto que a maior parte de seus estudos são submetidos a animais em fase de estudos pré-clínicos.

\section{Chapéu-de-couro (Echinodorus macrophyllus)}

O chapéu-de-couro (Echinodorus macrophyllus) é uma planta originária do sul do Brasil, e dispersa-se por quase todo território brasileiro. Pertence a família Alismataceae e ao gênero Echinodorus, sendo encontrado como Echinodorus macrophyllus e Echinodorus gandiflorus, tendo atividades terapêuticas semelhantes. Ambas possuem folhas longamente pecioladas e adapta-se bem em locais úmidos e sombreados, como várzeas, margens de rios e lagos (FRANCO et al., 2016).

Esta planta possui grande expressão na medicina popular, porém ainda com poucas informações sobre cultivo, variedades e métodos de conservação da planta (BEVILAQUA et al., 2001).

O uso desta planta como um fitoterápico inclui propriedades importantes como ação anti-inflamatória, antimicrobiana, depurativa, diurética e anti-hipertensiva, sendo apontada em vários estudos com animais. Os principais constituintes químicos encontrados nessa família são os alcalóides, flavonoides, taninos, triterpenos, glicosídeos, equinodorosídeos e sais minerais, e seu principio ativo é um diperpeno chamado de "echinodol" ou ácido echinóico, que é um metabólito secundário, obtido através de extração metanólica de folhas secas da planta, submetida á baixas temperaturas. (MARTINS, CASTELLANI, DIAS, 1995; CARDOSO, PEREIRA, LAINETTI, 2003). 
Em seu estudo Lessa et al., (2008), injetou doses de (300mg-1000mg/Kg) de extratos etanólicos de E. gandiflorusem ratos hipertensos, sendo observados efeitos anti-hipertensivos significante em comparação com drogas anti-hipertensivas utilizados como referencia em tratamento da hipertensão.

Uma pesquisa realizada por Conceição (2011) investigou os possíveis efeitos do extrato hidro alcoólico de E. gandiflorus sobre a hipertensão arterial e sobre a alteração micro circulatória de ratos espontaneamente hipertensos. Com as folhas da planta, por meio de maceração dinâmica obteve-se o extrato hidro alcoólico. Os animais, ratos machos adultos da linhagem Okamoto e Wistar Kyoto, foram divididos de maneira aleatória em 4 grupos, sendo um grupo tratado durante 28 dias por gavagem, grupo controle e um, com extrato hidro alcóolico de Echinodorusgrandiflorus (EG, $50 \mathrm{mg} / \mathrm{kg} / \mathrm{dia}, \mathrm{EG}, 100 \mathrm{mg} / \mathrm{kg} / \mathrm{dia}$ e EG, 200 $\mathrm{mg} / \mathrm{kg} / \mathrm{dia})$. Outro grupo de 10 ratos WKY foi tratado com veículo durante 28 dias e foi considerado como o grupo controle normotenso. Posteriormente foi aferida a pressão arterial sistólica e diastólica nos animais. Ao final do tratamento foi possível observar que o grupo tratado com as diferentes doses estudadas foi capaz de normalizar a pressão arterial sistólica quando comparados com os normotensos. Já o tratamento dos ratos WKY e SHR com veículo não se observou nenhuma alteração nos valores.

De acordo com a Agencia Nacional de Vigilância Sanitária - ANVISA (2011), essa planta deve ser utilizada como infusão, onde são utilizados 1 grama de suas folhas secas, para $150 \mathrm{ml}$ de água

\section{Conclusão}

A fitoterapia já é muito utilizada pela população em geral, através de conhecimentos, muitas vezes de senso comum, adquiridos de geração para geração, porém sem fundo cientifico. Muitos destes fitoterápicos já utilizados foram cientificamente testados e comprovados sua eficiência em determinadas enfermidades, incluindo doenças crônicas não transmissíveis.

Por meio desse estudo, foram analisados alguns fitoterápicos que atuam auxiliando na redução da hipertensão arterial, uma doença crônica que atinge grande parte da população. 
Algumas das plantas estudadas possuem esse efeito comprovado, porém muitos que são utilizados, ainda não foram testados em ensaios pré-clínicos e clínicos, não tendo seus efeitos comprovados.Desta forma, ainda são necessários outros estudos a fím de comprovar a eficiência de muitos fitoterápicos já utilizados no tratamento de doenças crônicas não transmissíveis.

\section{Referências}

ALBUQUERQUE, E.S.B.; NEVES, L.J. Anatomia foliar de Alpiniazerumbet (Pers.) Burtt \& Smith (Zingiberaceae). Acta Botanica Brasileira, v.18, p.109-21, 2004.

ALMEIDA, Â.; SUYENAGA, E.S. Ação farmacológica do alho (Alliumsativum L.) e da cebola (Allium cepa L.) sobre o sistema cardiovascular: revisão. Nutrire Revista da Sociedade Brasileira de Alimentação e Nutrição, v.34, p.185-197, 2009.

ALMEIDA, C. E. et al. Analysis of antidiarrhoeic effect of plants used in popular medicine. Revista de Saúde Publica, v. 29, n. 6, p. 428-433, 1995.

ANVISA; Agencia Nacional de Vigilância Sanitária. Farmacopéia Brasileira. $5^{\text {a }}$ edição. Brasília, 2011.

BEVILAQUA, G.A.; NEDEL, J.L.; CORREA, J.A.Z. Distribuição Geográfica E Composição Química De Chapéu De Couro (Echinodorus Spp.) No Rio Grande Do Sul. Ciência Rural, v. 31, n. 2, p. 213-218, 2001.

BEZERRA, J.E.F. et al. Comportamento da pitangueira (Eugenia uniflora L.) sob irrigação na região do Vale do Rio Moxotó, Pernambuco. Revista Brasileira de Fruticultura, Jaboticabal, v.26, n.1, p.177-179, 2004.

BRASIL. Ministério da Saúde. Portaria n ${ }^{\circ} 971$ de 03 de maio de 2006. Política Nacional de Práticas Integrativas e Complementares (PNPICS) no Sistema Único de Saúde, 2006.

CARDOSO, G.L.C.; PEREIRA, N.A.; LAINETTI, R. Avaliação das atividades antinociceptiva, antiinflamatória e diurética de chapéu de couro (Echinodorusgrandiflorus,[Cham. eSchl.] Mitch. Alismataceae). Revista Brasileira de Farmacologia, v. 84, p. 5-7, 2003.

CIRQUEIRA, R. T.; ALVES, M. J. Q. F. Efeitos hipotensivo e diurético dos extratos aquosos de pitanga (Eugenia uniflora L.) e jambolão (Eugenia jambolana Lam.) em ratos normotensos anestesiados. Revista Brasileira de Plantas Medicinais, p. 86-91, 2005. 
CONCEIÇÃO, F.G. et al. Efeitos anti-hipertensivos e microcirculatórios do extrato hidroalcóolico de Echinodorusgrandiflorus (chapéu de couro) em ratos espontaneamente hipertensos. Dissertação (Mestrado) - Instituto Oswaldo Cruz, Pós-Graduação em Biologia Celular e Molecular. Rio de Janeiro, 2011.

CONSOLINI, A. E.; BALDINI, O.A.N.; AMAT, A.G. Pharmacologicalbasis for theempirical use of Eugenia uniflora L.(Myrtaceae) as antihypertensive. Journal of Ethnopharmacology, v. 66, n. 1, p. 33-39, 1999.

COSTA, D.P. et al. Influence of fruit biotypes on the chemical composition and antifungal activity of the essential oils of Eugenia uniflora leaves. Journal of the Brazilian Chemical Society, v. 21, n. 5, p. 851-858, 2010.

DE QUEIROZ, J.M.G. et al. Aspectos populares e científicos do uso de espécies de Eugenia como fitoterápico. Revista Fitos Eletrônica, v. 9, n. 2, p. 87-100, 2015.

EMILIANO, A.F. Efeito vasodilatador do extrato hidroalcóolico da Alpiniazerumbet (Pers.) Burtt e Smith no leito vascular mesentérico. URJ -Rio de Janeiro, 2002.

FRANCO, R.M. et al. Ação antimicrobiana da planta chapéu-de-couro (Echinodorusmacrophyllus). Jovens estudantes no ambiente científico: resultados das pesquisas BIC-JR, da Universidade do Vale do Sapucaí, p. 33, 2016.

GUYTON, A.; HALL, J. Tratado De Fisiologia Médica. $11^{\mathrm{a}}$ edição. São Paulo: Elsevier, 2006.

LAHLOU, S., INTERAMINENSE, L. F. L., LEAL-CARDOSO, J. H., \& DUARTE, G. P. Antihypertensive effects of the essential oil of Alpiniazerumbet and its main constituent, terpinen-4-ol, in DOCA-salt hypertensive conscious rats. Fundamental \& clinical pharmacology, v.17, n.3, p.323-330, 2003.

LAMARÃO, R.; NAVARRO, F. Aspectos nutricionais promotores e protetores das doenças cardiovasculares. RBONE-Revista Brasileira de Obesidade, Nutrição e Emagrecimento, v. 1, n. 4, 2012.

LESSA MA, ARAUJO CV, KAPLAN MA, PIMENTA D, FIGUEIREDO MR, TIBIRICA E. Antihypertensive effects of crude extracts from leaves of Echinodorusgrandiflorus. Fundamental \& Clinical Pharmacology, v.22, n.2, p.161-8, 2008.

LORDELO, I.G.S. et al. Avaliação clínica e toxicológica da Alpiniazerumbet(Pers.) Brustt\& Smith em pacientes com hipertensão arterial estágio I (leve) ou estágio II (moderada). Sociedade Brasileira de Plantas Medicinais, v.16, 2000.

LORENZI, H; ABREU MATOS F. J. Plantas medicinais no brasil: Nativas e exóticas. 2. Ed. Nova Odessa, SP: Instituto Plantarum, 2008. 
MAGINA, M.A. et al. Atividade antioxidante de três espécies de Eugenia (Myrtaceae). Latin American Journal of Pharmacy, v. 29, n. 3, p. 376-82, 2010.

MAHAN, L.K.; SCOTT-STUMP, S. Krause: Alimentos, Nutrição e Dietoterapia. 11 edição. São Paulo: Elsevier, 2011.

MARMITT, D. J. et al. Revisión sistemática de las plantas de interés para el Sistema de Saludcon potencial terapéutico cardiovascular. Revista Cubana de Plantas Medicinales, v.21, p.108-124, 2016.

MARTINS E.R.; CASTELlANI,J.; DIAS, E. Plantas medicinais. Viçosa: UFV, v. 220, 1995.

MELLO, M.; BUDEL, J.M. Equisetum L.(Equisetaceae): uma revisão. Saúde, v.1, n.9, 2014. MENDONÇA, V. L. M.; OLIVEIRA, C. L. A.; CRAVEIRO, A. A. Pharmacologica land toxicológica levaluation of Alpinia speciosa. Memórias do Instituto Oswaldo Cruz, v.86, p.93-97, 1991.

MPALANTINOS, M.A.; MOURA, R.S.; PARENTE, P.; KUSTER, R. M. Biologically active flavonoids and kava pyrones from the aqueous extract of Alpinia zerumbet. Phytotherapy Research, v.12, p.442-4, 1998.

NOGUEIRA JUNIOR, J.C. Hipertensão arterial sistêmica: um estudo sobre a ótica ocidental e oriental e o uso de fitoterapia e ervas medicinais no Brasil. Monografia de especialização. Centro de Estudos Fivral: São José dos Campos, 2014.

PESSINI, G. L. et al. Avaliação da atividade antibacteriana e antifúngica de extratos de plantas utilizados na medicina popular. Revista Brasileira de Farmacognosia, v. 13, p. 2124, 2003.

SOUZA, L.K.H. et al. Antifungal properties of Brazilian Cerrado plants. Brazilian Journal of Microbiology, v. 33, n. 3, p.247-249, 2002.

TALBOTT, S. M.; HUGHES, K. Suplementos dietéticos para profissionais de saúde. Rio de Janeiro: Guanabara Koogan, 2015.

Como citar este artigo (Formato ABNT):

MEIRA, Elisangela de; KLOSTER, Edirléia de F.; KOSAK, Josieli M.; KOLITSKI, Mayara F.; CUTILAKI, Vinicius B.; MAZUR, Caryna E. O Uso de Fitoterápicos na Redução e no Tratamento de Hipertensão Arterial Sistêmica. Id on Line Revista Multidisciplinar e de Psicologia, 2017, vol.11, n.37, p.27-36. ISSN: 19811179 .

Recebido: 26.07.2017

Aceito: 08.08 .2017 